\title{
Probing the particle acceleration at trans-relativistic shocks with gamma-ray burst afterglows
}

\author{
Kazuya Takahashi, ${ }^{a, *}$ Kunihito loka, ${ }^{a}{ }^{\text {Yutaka Ohira }}{ }^{b}$ and Hendrik van Eerten ${ }^{c}$ \\ ${ }^{a}$ Center for Gravitational Physics, Yukawa Institute for Theoretical Physics, Kyoto University \\ Sakyo-ku Kitashirakawa Oiwakecho, Kyoto, Japan \\ ${ }^{b}$ The University of Tokyo, Department of Earth and Planetary Science \\ Bunkyo-ku, Tokyo, Japan \\ ${ }^{c}$ University of Bath, Department of Physics \\ Claverton Down, Bath BA2 7AY, United Kingdom \\ E-mail: kazuya.takahashi@yukawa.kyoto-u.ac.jp
}

The detail of the particle acceleration at trans-relativistic shocks is still under debate. We propose a way to probe the particle acceleration at trans-relativistic shocks with observations of gamma-ray burst (GRB) afterglows. In the afterglow phase, the shock wave launched in a GRB is gradually decelerated from the relativistic to non-relativistic regimes by sweeping up the ambient interstellar matter. If the electron power-law index depends on the shock Lorentz factor, it is reflected to the evolution of the afterglow spectrum. We theoretically study the time evolution of the electron power-law index imprinted in GRB afterglow spectra. We introduce a particle acceleration model by a trans-relativistic shock into the standard GRB afterglow model and apply the formulation to structured jet models that are consistent with GRB 170817A, which is the counterpart of the gravitational-wave signal GW170817 from a binary neutron star merger. As a result, we find that it is possible to observe the transition of the electron acceleration from the relativistic phase to the non-relativistic phase in the evolution of the afterglow spectrum, if GRBs similar to GRB 170817A take place in a dense environment at $200 \mathrm{Mpc}$. The detection number of short GRBs will increase in the era of the multi-messenger astronomy including gravitational waves. Thus, we expect that future GRBs can give a constraint on particle acceleration models as proposed in our study.

$37^{\text {th }}$ International Cosmic Ray Conference (ICRC 2021)

July 12th - 23rd, 2021

Online - Berlin, Germany

\footnotetext{
${ }^{*}$ Presenter
} 


\section{Introduction}

Particle acceleration across trans-relativistic shock waves has not been understood well. The value or time evolution of the electron power-law index $p$ is one of the unresolved issues. A transrelativistic shock wave is expected for gamma-ray burst (GRB) jets in the afterglow phase, where the shock wave gradually slows down from the relativistic to non-relativistic speeds by sweeping the ambient interstellar matter. The electron power-law index is imprinted in the GRB afterglow spectrum via the synchrotron radiation from the accelerated electrons [21]. Thus, GRBs can be a potential source that constrains the mechanism of particle acceleration at trans-relativistic shocks.

Recently, the afterglow spectrum for GRB 170817A, which is the counterpart of the gravitational wave signal GW170817 from a binary neutron star merger, is found to be almost constant with $p \sim 2.17[7,9,16]$. However, it could be a fortunate coincidence that the $p$ value measured in GRB 170817A falls within the range predicted in theory. Indeed, a single universal behaviour for $p$ is not manifested in other GRBs and SNRs $[4,23,29]$. Thus, the value or time evolution of $p$ in nature remains controversial.

In this paper, we study the off-axis GRB afterglows with a relativistic particle acceleration model. We show that the time evolution of the electron power-law index $p$ can be observable in future off-axis GRB afterglows if the GRB takes place in a dense environment. The observed evolution of $p$ could constrain the particle acceleration models.

Throughout the paper, we attach a prime to the quantities evaluated in the fluid rest frame. The quantities without a prime are evaluated in the laboratory frame unless otherwise mentioned.

\section{Method}

We assume a particle acceleration model that takes into account the relativistic effects and apply it to the calculation of GRB afterglows. We consider off-axis GRB afterglows (i.e., the line of sight is not parallel to the jet axis), since the detection number of the nearby off-axis events would increase in the era of the multi-messenger astronomy including gravitational waves. We take into account the jet structure of GRB jets, since the jet structure is important for off-axis afterglows as the uniform top-hat jets are rejected for GRB 170817A [17]. We review the off-axis afterglow model in Section 2.1. Then, we present the model of the structured jet in Section 2.2, which determines the shock evolution.

\subsection{Off-axis afterglow model}

We theoretically reproduce an off-axis afterglow light curve and the spectrum by giving a jet structure and afterglow parameters. We apply the standard model of GRB afterglows by Sari et al. [21] to calculate the observed off-axis afterglow flux, where the afterglow is produced by the synchrotron radiation emitted from the non-thermal electrons accelerated across the forward shock. In the standard model, the energy spectrum of the accelerated electrons is given by a single power law with a constant power law index $p$. This study takes into account the dependence of $p$ on the shock Lorentz factor $\Gamma_{\text {sh }}$. We apply the model of Keshet \& Waxman [13] as an example. Microscopic physics such as the amplification of magnetic fields and particle acceleration through the shock wave is modelled by introducing phenomenological parameters, $\varepsilon_{\mathrm{B}}$ and $\varepsilon_{\mathrm{e}}$, respectively. 


\subsubsection{Evolution of the shock wave}

The evolution of the blast wave is given as same as in Takahashi \& Ioka [25, 26] (hereafter TI20 and TI21, respectively). The jet is assumed to be axi-symmetric and adiabatically propagates into a stationary, cold, uniform medium with a number density $n_{0}$. The jet has an angular structure that the isotropic equivalent energy is given as $E(\theta)$, where $\theta$ is the polar angle measured from the jet axis. We assume that each jet segment spherically expands as if it were a portion of the isotropic blast wave with the same isotropic equivalent energy. This assumption holds well for a relativistic shock unless it is decelerated sufficiently below a local sound speed and each segment interacts with each other $[14,30,32]$. Then, the dynamics of each segment of the shock wave is described by a self-similar solution of Blandford \& McKee [2]. As the shock slows down to a non-relativistic speed by sweeping up the ambient matter, the shock dynamics is better described by the Sedov-Taylor solution [22, 27]. We smoothly connect the Blandford-McKee and Sedov-Taylor solutions by giving the shock dynamics as follows [31]:

$$
\Gamma_{\mathrm{sh}}^{2} \beta_{\mathrm{sh}}^{2}=C_{\mathrm{BM}}^{2} t^{-3}+C_{\mathrm{ST}}^{2} t^{-6 / 5}
$$

where $\beta_{\mathrm{sh}}$ is the shock speed divided by the speed of light $c$, which is measured in the laboratory frame, $\Gamma_{\mathrm{sh}}$ is the shock Lorentz factor, and $t$ denotes the elapsed laboratory time since the explosion. The coefficients $C_{\mathrm{BM}}$ and $C_{\mathrm{ST}}$ are respectively given by $C_{\mathrm{BM}}=\sqrt{17 E /\left(8 \pi n_{0} m_{\mathrm{p}} c^{5}\right)}$ and $C_{\mathrm{ST}}=$ $\left[E /\left(n_{0} m_{\mathrm{p}} c^{5}\right)\right]^{1 / 5} \cdot 1.15 \cdot 2 / 5$, where $m_{\mathrm{p}}$ stands for the proton mass. The radius of each shock segment at a laboratory time, $R(t)$, is given by integrating the shock speed $c \beta_{\mathrm{sh}}$ from $t=0$ to $t$.

\subsubsection{Shock downstream quantities and electron power-law index $p$}

The physical quantities in the downstream flow just below the shock wave are determined from the Rankine-Hugoniot conditions for $\Gamma_{\text {sh }}$ given by Equation (1) [2] We solve the shock jump conditions with the Jüttner-Synge equation of state $(\operatorname{EoS})[3,12,24]$, which follows from the isotropic equilibrium state of a non-degenerate gas by taking into account the relativistic effects. The strength of the downstream magnetic field is phenomenologically given by $B^{\prime}=\sqrt{8 \pi \varepsilon_{\mathrm{B}} e_{\mathrm{i}}^{\prime}}$, where $\varepsilon_{\mathrm{B}}$ is the energy conversion fraction from the shocked matter to the magnetic field and $e_{\mathrm{i}}^{\prime}$ the downstream internal energy density.

The electrons are assumed to be accelerated across the shock wave and possess an isotropic energy spectrum given by a single power law with an index $p$. Then, the electron energy distribution function $N_{\mathrm{e}}^{\prime}\left(E_{\mathrm{e}}^{\prime}\right)$ is given by $N_{\mathrm{e}}^{\prime}\left(E_{\mathrm{e}}^{\prime}\right) \propto \gamma_{\mathrm{e}}^{\prime-p}$ for $\gamma_{\mathrm{e}}^{\prime} \geq \gamma_{\mathrm{m}}^{\prime}$, where $\gamma_{\mathrm{e}}^{\prime}$ is the Lorentz factor of an accelerated electron and $\gamma_{\mathrm{m}}^{\prime}$ is the minimal Lorentz factor of the non-thermal electrons [21]. $\gamma_{\mathrm{m}}^{\prime}$ and the normalization factor are determined from the conservation laws of mass and energy. The energy of the accelerated electron is assumed to be a fraction of $\varepsilon_{\mathrm{e}}$ of the internal energy of the shocked matter.

We assume that the electron power-law index $p$ depends on the shock speed. In this study, we employ the model of Keshet \& Waxman [13] as an example, who considered the diffusive shock acceleration across a relativistic shock wave with an isotropic diffusion. Then, the electron power-law index is given by

$$
p=\frac{3 \beta_{\mathrm{u}}-2 \beta_{\mathrm{u}} \beta_{\mathrm{d}}^{2}+\beta_{\mathrm{d}}^{3}}{\beta_{\mathrm{u}}-\beta_{\mathrm{d}}}-2,
$$


where $\beta_{\mathrm{u}}$ and $\beta_{\mathrm{d}}$ are respectively the upstream and downstream fluid speeds measured in the shock rest frame in unit of $c$. Through the Lorentz transformation, we find $\beta_{\mathrm{u}}=\beta_{\mathrm{sh}}$ and $\beta_{\mathrm{d}}=$ $\left(\beta_{\mathrm{sh}}-\beta\right) /\left(1-\beta \beta_{\mathrm{sh}}\right)$, where $c \beta$ is the speed of the shocked fluid measured at the laboratory frame. Note that $\beta$ is obtained by solving the shock jump conditions and, hence, is a function of $\Gamma_{\text {sh }}$. We also note that the value of $p$ is different among the jet segments, since $\Gamma_{\text {sh }}$ evolves in different ways for each jet segment, depending on the jet structure $E(\theta)$. Equation (2) yields $p=2$ in the non-relativistic limit $\left(\Gamma_{\mathrm{sh}} \rightarrow 1\right)$ and $p=20 / 9 \sim 2.22$ in the relativistic limit $\left(\Gamma_{\mathrm{sh}} \gg 1\right)$ with the Jüttner-Synge EoS [13].

\subsubsection{Observed afterglow flux}

The observed flux of the afterglow is calculated by integrating the synchrotron radiation emitted from each position on the jet surface. Under the thin-shell approximation, the observed synchrotron flux is given by [31]

$$
F_{v}(T)=\left.\frac{1}{4 \pi D^{2}} \int_{0}^{\theta_{\mathrm{j}}} \mathrm{d} \theta \int_{0}^{2 \pi} \mathrm{d} \varphi \frac{\mu R^{2} \sin \theta}{\Gamma^{3}(1-\beta \mu)^{3}} \frac{\epsilon_{v^{\prime}}^{\prime}}{\alpha_{v^{\prime}}^{\prime}}\left(1-e^{-\tau_{v}}\right)\right|_{t=T+\mu R / c},
$$

where $D$ is the luminosity distance to the source and we neglect the effect of the redshift for simplicity. $\theta_{\mathrm{j}}$ is the jet truncation angle. $\epsilon_{v^{\prime}}^{\prime}$ is the energy radiated by synchrotron emission per unit volume per unit time per unit frequency and $\alpha_{v^{\prime}}^{\prime}$ is the absorption coefficient for synchrotron self-absorption. The factor $1 /\left[\Gamma^{3}(1-\beta \mu)^{3}\right]$ comes from the Lorentz transformation of $\epsilon_{v} / \alpha_{v}$. The integrand is evaluated at the laboratory time $t=T+\mu R / c$, when the emitted photons reach the observer at an observer time $T$, where $\mu$ is the cosine of the angle between the radial direction and the line of sight (i.e., $\mu$ depends on the viewing angle $\theta_{\mathrm{v}}$ ).

Note that the shape of the local synchrotron spectrum depends on the electron power-law index $p$ given by Equation (2) and, as a result, the shape of the observed afterglow spectrum also depends on $p$ and evolves with time. The local synchrotron emissivity $\epsilon_{v^{\prime}}^{\prime}$ is given by a broken power-law: $\epsilon_{v^{\prime}}^{\prime} \propto v^{\prime 1 / 3}\left(v^{\prime}<v_{\mathrm{m}}^{\prime}\right), \epsilon_{v^{\prime}}^{\prime} \propto v^{\prime-(p-1) / 2}\left(v_{\mathrm{m}}^{\prime}<v^{\prime}<v_{\mathrm{c}}^{\prime}\right)$, and $\epsilon_{v^{\prime}}^{\prime} \propto v^{\prime-p / 2}\left(v_{\mathrm{c}}^{\prime}<v^{\prime}\right)$ for the slow cooling case, where $v_{\mathrm{m}}^{\prime}$ and $v_{\mathrm{c}}^{\prime}$ are the synchrotron characteristic frequency and cooling frequency, respectively [21].

The afterglow flux for a given frequency $v$ is generated by Equation (3) after specifying the parameters $\left\{n_{0}, \varepsilon_{\mathrm{B}}, \varepsilon_{\mathrm{e}}, \theta_{\mathrm{v}}, \theta_{\mathrm{j}}, D\right\}$ and a jet structure $E(\theta)$.

\subsection{Model of structured jets}

We apply the off-axis GRB afterglow model described in Section 2.1 to structured jet models. The jet structure is important for off-axis GRBs as the uniform top-hat jets cannot explain the slow rise of the afterglow of GRB 170817A [17]. As demonstrated in [TI20, TI21], there are many possible candidates of the jet structure that is consistent with the observed afterglow within observational errors. In this study, we pick up three examples of candidate jet structures shown in Figure 1 for examples: hollow-cone, Gaussian, and spindle jets. They generate consistent afterglow light curves at least for a constant power-law index $p=2.17$ [TI21]. As shown in Section 3, these jets are still consistent with the observations even if the electron power-law index $p$ is evolved with Equation (2). 


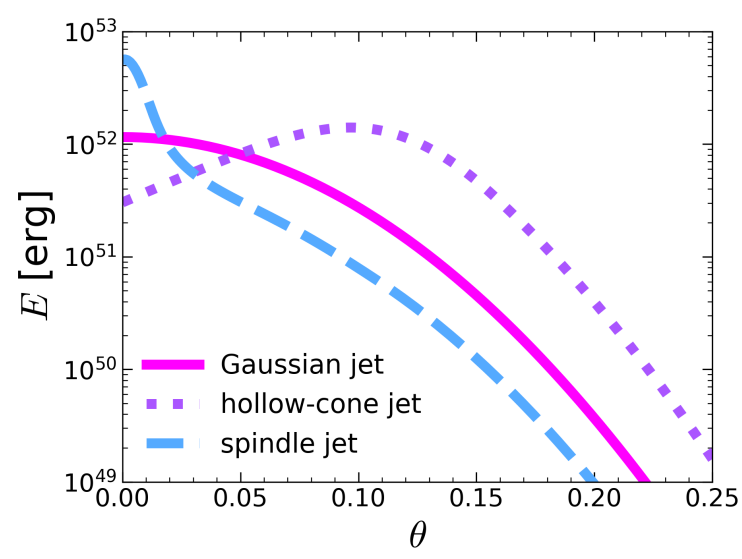

Figure 1: Three kinds of the jet structures considered in this study, which are referred from figure 1 in TI21: a hollow-cone jet (dotted line), a Gaussian jet (solid line), and a spindle jet (dashed line). $E(\theta)$ is the isotropic equivalent energy and $\theta$ is the angle measured from the jet axis. They are consistent with the afterglow of GRB 170817A as shown in TI21.

\section{Results}

We study the evolution of the electron power-law index $p$ imprinted in the afterglow spectrum. In Section 3.1, we show that the particle acceleration model given by Equation (2) is consistent with the observations of the afterglow of GRB 170817A. We also show that the time evolution of $p$ is insensitive to the jet structures. In Section 3.2, we study the evolution of $p$ by using a larger value for the ambient matter density $n_{0}$, expecting future binary neutron star merger events in a dense environment. We show that the transition of the electron power-law index from the relativistic to non-relativistic regimes can be observable for future events.

\subsection{Evolution of the electron power-law index for GRB 170817A}

Figure 2 shows the results of the time evolution of the electron power-law index imprinted in the afterglow (left) and the corresponding afterglow light curves (right). As shown in the figure, these results are consistent with the observations within the observational errors. Hence, the particle acceleration model given by Equation (2) and the jet structures shown in Figure 2 are consistent with the afterglow of GRB 170817A. Here, we used the fiducial values for the model parameters that are adopted in TI21: $n_{0}=10^{-3} \mathrm{~cm}^{-3}, \theta_{\mathrm{v}}=0.387 \sim 22.2^{\circ}, \varepsilon_{\mathrm{e}}=0.1, \theta_{\mathrm{j}}=0.61 \sim 35.0^{\circ}$, and $D=41 \mathrm{Mpc}$, while $\varepsilon_{\mathrm{B}}$ is tuned for each jet structure as $\varepsilon_{\mathrm{B}}=2.44 \times 10^{-4}$ for the Gaussian jet, $\varepsilon_{\mathrm{B}}=4.12 \times 10^{-5}$ for the hollow-cone jet, and $\varepsilon_{\mathrm{B}}=6.60 \times 10^{-4}$ for the spindle jet.

\subsection{Evolution of the electron power-law index for GRBs in a dense ambient medium}

Figure 3 shows the results for a denser environment with $n_{0}=1 \mathrm{~cm}^{-3}$ and farther distance $D=200 \mathrm{Mpc}$ (the other model parameters are unchanged from those in Section 3.1). The transition of the electron power-law index $p$ from the relativistic to non-relativistic regimes will be more accurately observable than in GRB 170817A, thanks to the more luminous light curves under the denser environment. 

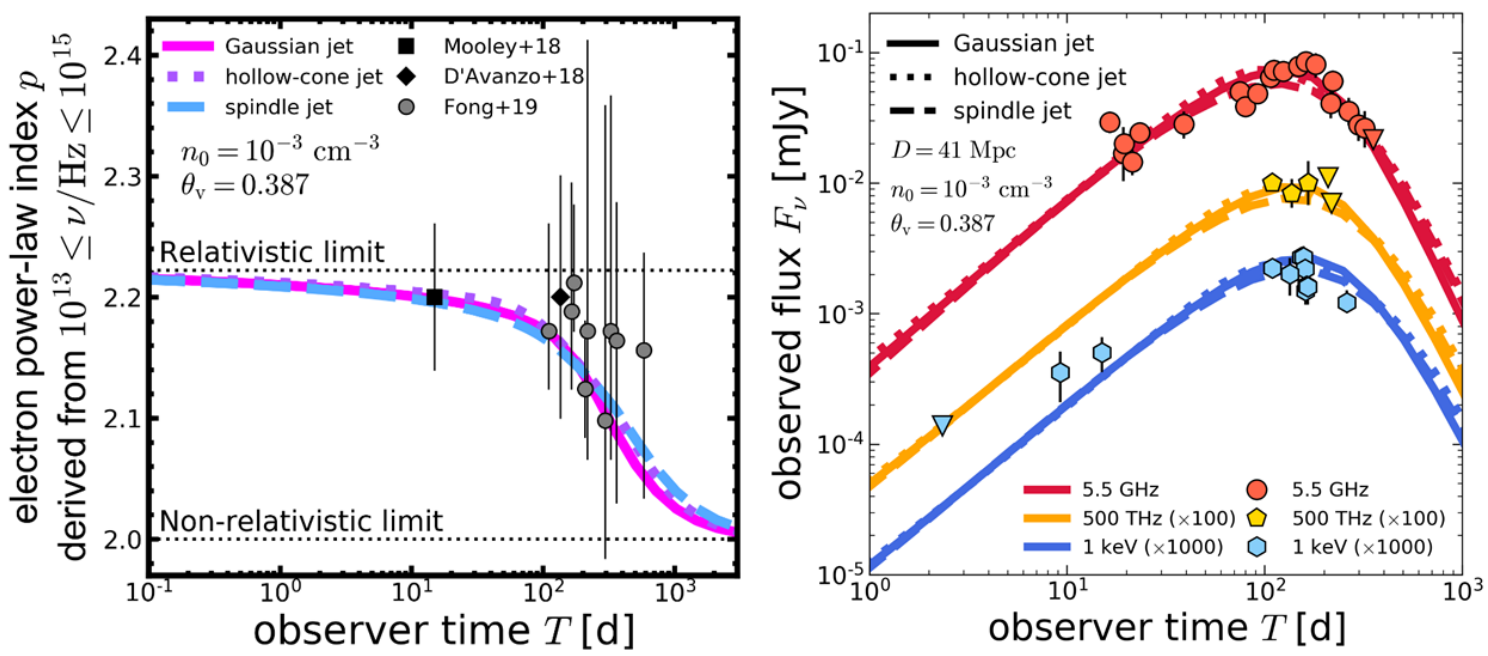

Figure 2: Left: Evolution of the electron power-law index $p$ for various jet structures. The electron power-law index is derived from the slope of the afterglow spectrum by assuming $F_{v} \propto v^{-(p-1) / 2}$ for $10^{13} \leq v / \mathrm{Hz} \leq 10^{15}$. The upper and lower dotted lines show the relativistic limit $(p=2.22)$ and the non-relativistic limit $(p=2)$ of the diffusive shock acceleration model given by Equation (2). Also plotted are the observed electron power-law index in GRB 170817A that are derived from multi-band spectra. The square-shaped and diamond-shaped plots are referred from Mooley et al. [17] and D'Avanzo et al. [6], respectively. The round-shaped plots are referred from table 2 in Fong et al. [9], which uses the data in $[1,7,10,16,17,19,20,29]$. Uncertainties correspond to $1 \sigma$. Right: Afterglow light curves of the structured jets with the electron power-law index $p$ that depends on the shock Lorentz factor as given by Equation (2). The solid, dotted, and dashed lines show the light curves of the Gaussian, hollow-cone, and spindle jets, respectively. Each colour corresponds to the radio $(5.5 \mathrm{GHz}$, red), optical $(500 \mathrm{THz}$, orange), and X-ray ( $1 \mathrm{keV}$, blue). Also plotted are the observed afterglow fluxes (points) and upper limits (lower triangles) of GRB 170817A. The data points for radio were taken from Figure 4 in Troja et al. [29], which uses the data in $[1,11,15-17,20,28]$. The data points for optical and X-ray were collected from $[1,6,15,16,20]$.

The left panel of Figure 3 shows that the timescale of the evolution of the electron power-law index is smaller than for a smaller $n_{0}$ (cf. Figure 2). For example, the transition from the relativistic to non-relativistic limits takes place at earlier time, $T \sim 10-100 \mathrm{~d}$. This is because the shock is decelerated more effectively due to the denser ambient medium. Again, the time evolution is insensitive to the jet structures.

The right panel shows that the afterglow light curves of the Gaussian jet can be more luminous than for GRB 170817A. The afterglow flux becomes larger just because the denser environment, in spite of the larger distance. In the shaded area, the afterglow flux is larger than that in GRB 170817A and the expected kilonova flux in the optical band, where the electron power-law index is imprinted in the spectrum. Hence, the electron power-law index $p$ would be more accurately obtained in the shaded time window than in GRB 170817A. Importantly, this time window overlaps with the time window when the electron power-law index rapidly transits from the relativistic regime to the non-relativistic regime (cf. the left panel).

The above result is qualitatively the same for the hollow-cone and spindle jets. The result is also qualitatively the same for other viewing angles. 

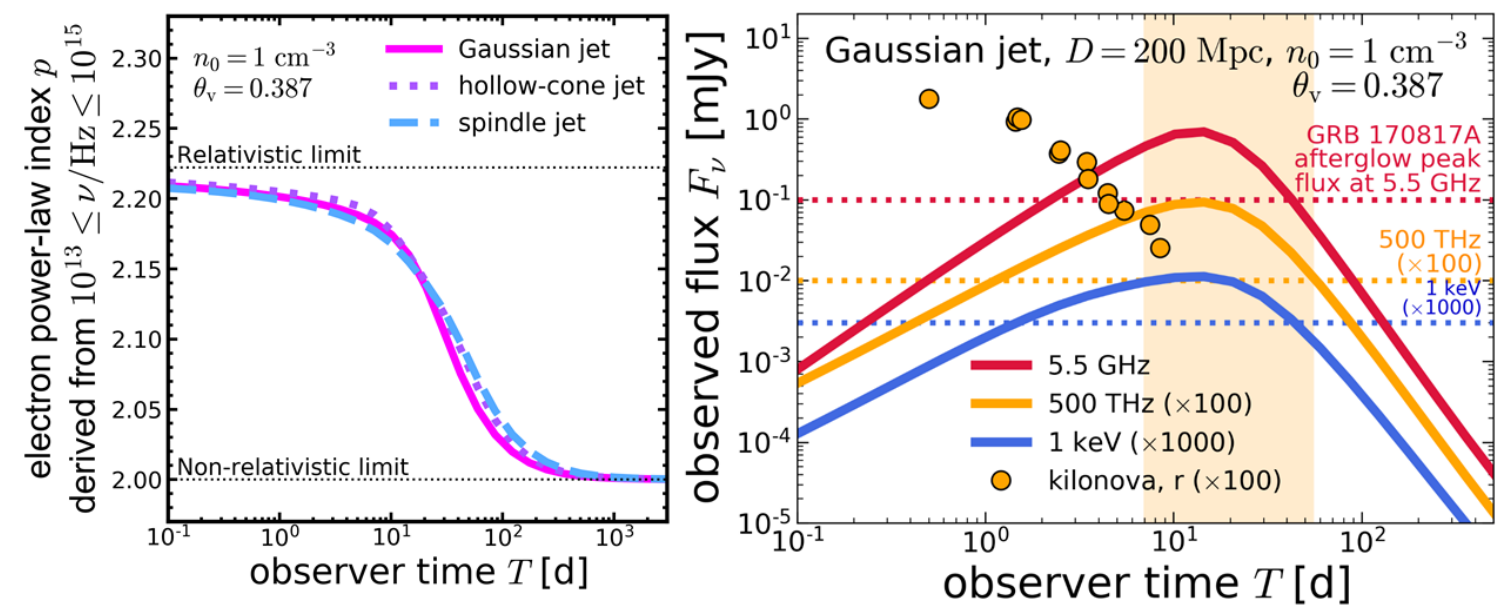

Figure 3: Left: Same as the left panel of Figure 2 but for $n_{0}=1 \mathrm{~cm}^{-3}$. The timescale of the evolution is smaller than for $n_{0}=10^{-3} \mathrm{~cm}^{-3}$, due to the smaller deceleration timescale of the forward shock. Right: The corresponding afterglow light curves for the Gaussian jet with $n_{0}=1 \mathrm{~cm}^{-3}$ and $D=200 \mathrm{Mpc}$. In the shade region, the optical afterglow is more luminous than the afterglow of GRB 170817A and the kilonova and, hence, the electron power-law index $p$ would be more accurately obtained. The afterglow flux can be more luminous because of the larger ambient density, in spite of the farther distance. The expected kilonova flux (round-shaped points) is obtained by converting the observed fluxes of the kilonova associated with GRB 170817A [5, 8] to the values at $D=200 \mathrm{Mpc}$.

\section{Conclusions}

As demonstrated in this study, the time evolution of the electron power-law index $p$ would be observable in the spectral evolution of future off-axis GRB afterglows in a dense environment. In principle, one can predict the spectral evolution with any particle acceleration model, whereas we employed the model of Keshet \& Waxman [13], where $p$ is given by Equation (2), as an example. Hence, the particle acceleration at trans-relativistic shocks could be probed by future GRB afterglow observations. A straightforward way to constrain the particle acceleration model is to plot the observed value of $p$ as a function of $\Gamma_{\mathrm{sh}} \beta_{\mathrm{sh}}$. The shock Lorentz factor can be obtained if the superluminal motion of the jet is detected with VLBI in the afterglow phase [18], for example. These issues will be discussed in our forthcoming paper.

\section{References}

[1] Alexander K. D. et al., 2018, ApJL, 863, 18

[2] Blandford R. D., McKee C. F., 1976, Phys. Fluids, 19(8), 1130

[3] Cercignani C., Kremer G. M., 2002, The relativistic Boltzmann equation: theory and applications, Springer, Basel

[4] Curran P. A., Evans P. A., de Pasquale M., Page M. J., van der Horst A. J., 2010, ApJL, 716, L135 
[5] Cowperthwaite P. S. et al., 2017, ApJL, 848, L17

[6] D’Avanzo P. et al., 2018, A\&A, 613, L1

[7] Dobie D. et al., 2018, ApJL, 858, L15

[8] Drout M. R. et al., 2017, Science, 358, 1570

[9] Fong W. et al., 2019, ApJL, 883, L1

[10] Hajela A. et al., 2019, ApJL, 886, L17

[11] Hallinan G. et al., 2017, Science, 358, 1579

[12] Jüttner F., 1911, AnP, 339, 856. doi:10.1002/andp.19113390503

[13] Keshet U., Waxman E., 2005, PRL, 94, 111102

[14] Kumar P., Granot J., 2003, ApJ, 591, 1075

[15] Lyman J. D. et al., 2018, NatAs, 2, 751

[16] Margutti R. et al., 2018, ApJL, 856, 18

[17] Mooley K. P. et al., 2018a, Nature, 554, 207

[18] Mooley K. P. et al., 2018b, Nature, 561, 355

[19] Mooley K. P. et al., 2018c, ApJL, 868, 11

[20] Piro L. et al., 2019, MNRAS, 483, 1912

[21] Sari R., Piran T., Narayan R., 1998, ApJL, 497, 17

[22] Sedov L. I., 1959, Similarity and Dimensional Methods in Mechanics. Academic Press, New York

[23] Shen R., Kumar P., Robinson E. L., 2006, MNRAS, 371, 1441

[24] Synge J. L., 1957, The Relativistic Gas, North-Holland Publishing Company, Amsterdam

[25] Takahashi K., Ioka K., 2020, MNRAS, 497, 1217

[26] Takahashi K., Ioka K., 2021, MNRAS, in press, arXiv:2007.13116

[27] Taylor G., 1950, Proc. R. Soc. A, 201, 159

[28] Troja E. et al., 2018, MNRAS, 478, L18

[29] Troja E. et al., 2019, MNRAS, 489, 1919

[30] van Eerten H. J., MacFadyen A. I., 2012, ApJ, 751, 155

[31] van Eerten H., Zhang G., MacFadyen A., 2010, ApJ, 722, 235

[32] Zhang W., MacFadyen A., 2009, ApJ, 698, 1261 